ARCH. INT. MED.

\title{
THE DISTRIBUTION OF BILE IN CERTAIN TYPES OF JAUNDICE *
}

\author{
M. A. BLANKENHORN, M.D. \\ CLEVELAND
}

In a previous publication from this clinic $^{1}$ it was proposed by me to examine the blood for bile in cases of jaundice, and a scheme devised to make measurements on blood, stool and urine which, when correlated with the color of the skin, might throw some light on the rôle of bile in the various types of jaundice. The work covered by this report is the examination of 395 specimens of blood plasma together with an examination of the urine and skin, and in a lesser number an examination of stool and duodenal contents, according to that scheme. The methods employed are essentially those previously described, but with the following modifications. A tintometer constructed in the laboratory is now used to measure the color of the plasma, and readings can be made more accurately than by our earlier method, by which test tubes were simply held parallel and observed down the length of the tube. The color of the plasma is taken as an index of the bilirubin content and the amount is expressed by a number corresponding to the number of times the plasma must be diluted to reduce the color to a tint that can just be discerned in a column $1 \mathrm{~cm}$. deep.

I am of the same opinion as formerly expressed, that the yellow color of plasma is due solely to bilirubin, and that Gmelin's test with nitric acid can be used to test for it. To substantiate this claim I made parallel tests in 239 cases, using Gmelin's and the Huppert-Cole tests. The results were identical in 224 cases, contradicting in 15 . In 3 , the Huppert-Cole was positive while the nitric acid test was negative. In 12 the nitric acid test was positive and the Huppert-Cole test negative. But the value of the Huppert-Cole tests depends to a certain extent on the quantity of plasma used, and it is sometimes negative because not enough plasma is available. This slight discrepancy is certainly explainable by the difference in delicacy of the tests and does not disprove at all the identity of the substance tested for.

The test for bile salts in the blood is modified so that we may now dialyze 3 c.c. of plasma in 9 c.c. of water through collodion sacs, where

* Submitted for publication Nov. 18, 1917.

* Read before the Association of American Physicians, May, 1917.

1. Blankenhorn, M. A.: The Bile Content of the Blood in Pernicious Anemia, The Archives Int. Med., 1917, 19, 344. 
formerly we dialyzed 5 c.c. into 10 c.c. of water and alcohol. For cholesterol in the blood I have found no satisfactory and practicable method.

Bile Pigment.-Three hundred and ninety-five specimens of blood plasma were examined in this manner. Of this number, seventy-five were described as colorless; that is, there was no distinct yellow color present, there being only a faint turbidity, or a faint pink or buff tint distinguishing the specimen from water. This group of seventy-five all gave negative results when tested chemically for bilirubin.

Two hundred and twenty-two specimens were distinctly yellow and gave a chemical test for bilirubin. The specimens of this group measured from 15 to 375 . Forty represents the critical point at which the cholemia becomes a visible jaundice. Of the 222 specimens, only two were over 40 without the patient being jaundiced, and but ten were under 40 with the patient jaundiced.

Between the 75 specimens and the 222 giving a chemical test for bilirubin were 94 specimens that were distinctly yellow in color, but gave no chemical test for bilirubin. It is in this group that the possibility of the presence of some yellow substance other than bilirubin must be seriously considered. In this group was the blood from 10 primary anemia patients; from 7 patients who had been jaundiced previously; from 11 with acute infectious diseases in which jaundice is very common; and 6 with some disease of the liver or gall ducts; that is, 34 , or about one third, in which bilirubin is commonly found. In some of them bilirubin had actually been found previously, and in others it was found subsequently. Inferentially, we can say that bilirubin was present in one third of all these, but in amounts too small to be detected chemically. The remaining two thirds were all from normal individuals or patients admitted to the hospital for some condition not associated with jaundice. In all this class of doubtful, subicteric plasmas, at no time was there ever present any pigment in large amounts. I have repeatedly tested those specimens for luetin and urobilin, which are supposed to pigment the plasma at times, but in this group neither of these was found. And since it is common to find bilirubin in amounts large enough to be tested chemically in perfectly normal persons, it is safe to assume that bilirubin is the pigment in this entire group of 94 .

By an analysis of the 222 specimens of blood plasma that contained bilirubin in amounts large enough to give a chemical test, considerable light is thrown on the behavior of bilirubin, per se. This has aided distinctly in the interpretation of the various findings in particular groups of cases and somewhat in the interpretation of individual cases. As indicated in the foregoing, the degree of staining of the plasma 
varied from 15 to 375 and 40 represents the critical point at which the cholemia becomes jaundice; 100 represents a distinct jaundice; 300 and over a marked jaundice. The series does not contain a case of that very extreme jaundice sometimes seen in prolonged, complete biliary obstruction.

One hundred and forty-one of the specimens were from jaundiced patients; 81 were from patients not jaundiced. In the group of 81 are 14 from normal patients; 20 from patients admitted for conditions in no way associated with jaundice. I frequently find blood plasma very distinctly stained with bilirubin where there is absolutely no explanation, unless it be that a relative degree of cholemia is normal. A goodly number of these cases were investigated repeatedly and the findings confirmed, but no individual or physiologic variation can be described as yet. On the other hand, the degree of cholemia was frequently found distinctly above 40 , the critical point where jaundice should be expected, yet none could be observed by the ward physicians. Three were found at 50 or over.

Of the 395 examinations made, it was found in seven cases that the patient was described as jaundiced, but the plasma was found absolutely colorless. This at first suggests jaundice without cholemia, but six out of seven cases were patients with a very severe secondary anemia, in which the normal pigmentation is exaggerated by pallor, and the condition simulates jaundice; the remaining one was described as doubtful. We thus dismiss from this report jaundice without demonstrable cholemia.

I have found that a distinctly higher degree of cholemia can exist without choluria, and 60 is found to express the critical point for choluria or the average above which cholemia becomes choluria. There is, however, a wide range in the degree of cholemia without choluria, so much so that when some of the more certain factors concerned in the behavior of bilirubin in the blood are determined, this disparity may be of much diagnostic importance.

One hundred and fifty-three of 220 specimens showed a chemical test for bilirubin in the plasma without choluria. These ranged from 15 to 275 . In many cases this retention of bilirubin was associated with impaired kidney function. In other cases no other retention could be demonstrated and there was no reason to expect any impairment of kidney function. The duration of the jaundice, as well as the intensity, seems to be a factor in determining this disparity. In general, a more prolonged and a more severe jaundice is associated with a high cholemia when there may be no choluria. Also, when there is disease of the liver with change in its size and consistency, this disparity is frequently marked. In diseases of the bile ducts the disparity is less 
frequent and less marked. Choluria without cholemia was not seen; also choluria without jaundice, in so far as bilirubin alone is concerned, has not been observed.

From the foregoing analysis of 222 cases in which bilirubin was found chemically, I am impressed with the following points in the behavior of bilirubin in the blood; that bilirubin is very commonly present in the blood of normal individuals in varying amounts; that it is usually readily diffused through the lymph into the skin, but less readily through the kidney; that it may be fixed in the blood, and thus withheld from lymph and urine; that it is not formed or retained in the lymph in higher concentration than in the blood; that it never appears in the urine without reaching and maintaining a certain concentration in the blood.

Bile Salts.-In studying the bile salts in the blood I have thus far found no satisfactory quantitative method. Further studies on the dialysis of blood plasma through collodion into water confirms the belief that cholesterin and lecithin are not so dialyzed, and that the Pettenkofer test on the dialysate, together with the spectrum, is a specific test for bile salts. It is not yet possible to bring much order out of the chaotic findings that appear in investigating bile salts, and until a quantitative method is used and a more satisfactory method is found for the urine, very little can be said with assurance about the behavior of bile salts in the blood. A brief analysis of some of the results alone is justifiable.

Out of 395 specimens examined, bile salts were found in 124 . In 87 cases bile salts occurred in the blood in conjunction with bilirubin. This occurs most commonly when bilirubin is highly concentrated in the blood and when both bile salts and bilirubin are present in the urine. In 37 cases bile salts occurred alone. This was most often in cases of primary anemia, acute infectious diseases, diseases of the liver in which there is change in the size and consistency of the liver, and in nephritis. In 10 cases bile salts were found in conditions never associated with jaundice. In 4 cases bile salts were found in normal individuals. When larger amounts of plasma are dialyzed, bile salts are very often found in normal blood, and it is my impression that if 15 c.c. of plasma are dialyzed by the method described and all the dialysate be concentrated into 2 c.c., the Pettenkofer test will be uniformly positive in normal persons. In 106 cases bile salts were found in the blood, and not in the urine; in 13 bile salts were in the urine and not in the blood. This disparity of salts between cholemia and choluria, with a very small number showing salts in the urine and none in the blood, but a large number with salts in the blood and none in the urine, suggests a process of retention; whether this is due to a 
fixation in the blood or a diminished permeability of the kidney is not yet clear. More accurate methods for examining the urine and a quantitative study of the blood are necessary to clear this up.

Urobilin.-One hundred and thirty-three specimens of blood were examined for urobilin and twenty-two found positive. This rather large number was at first surprising after the consistently negative results which I have previously reported and which other observers report. The method used is the same as that used previously, namely, the plasma is centrifuged with an equal volume of 90 per cent. alcohol saturated with zinc acetate and Ehrlich's aldehyd reagent added to the clear liquid. Both the purple red color and the spectrum were found in every positive test. The positive results are attributed to the selection of the patients and the time of taking the specimens.

Of the twenty-two positive cases, in eighteen urobilin occurred in conjunction with bilirubin, and in nine cases it was found where no jaundice was observed. In three instances urobilin was found in large amounts in the plasma of patients that were not jaundiced and moreover the plasma itself was practically colorless.

The urine was examined in 325 cases and urobilin found 110 times. In eighty-eight cases there was urobilin in the urine with none in the blood. In no instance was there urobilin in the blood, with none in the urine. In but two of the patients in whom a urobilinemia was found was there any distinct impairment of the kidney. In every case in which there was urobilinemia, enormous amounts were also found in the urine. It is, therefore, quite obvious that there is no retention of urobilin in the blood. Urobilin dialyzes very promptly into water through collodion membranes and leaves a perfectly clear and colorless dialysate; it can then be found in the dialysate by the Ehrlich aldehyd reagent and the spectroscope. From the foregoing observation we can say that urobilin is frequently found in the blood in large amounts without causing jaundice; that it is very promptly yielded to the kidney, and that it does not occur in the blood unless it is formed in excessive amounts.

We have over a thousand quantitative estimates of urobilin in stool and urine according to the methods of Wilbur and Addis $;^{2}$ but I do not feel that this has contributed anything to the general information on the distribution or behavior of bile. The difficulties due to irregularities in the formation and elimination of stool, catharsis, etc., have not yet been overcome; otherwise the method is entirely successful.

In the examination of the urine the methods used are the same as previously reported. No extensive use has been made of the quanti-

2. Wilbur and Addis: The Archives Int. Med., 1914, 13, 235. 
tative method for bilirubin, as described by Whipple. ${ }^{3}$ Although it has worked quite satisfactorily in deeply pigmented plasmas and urines, when smaller amounts of pigment are present $I$ have difficulty in getting readable colors.

In a previous publication by Hoover and myself, ${ }^{4}$ dissociated jaundice was elaborated and some of its terms defined. In the present report little can be added to the general premises of that work, especially anything on the significance of dissociation itself. In considering the various groups of cases separately the dissociation begins to take some form and meaning. Practically every case of jaundice, if the urine and blood be followed carefully through the onset and disappearance of the jaundice, will present dissociation at some time or other.

Laennec's Cirrhosis.-I have observed 18 cases of Laennec's cirrhosis with alteration in size and consistency of the liver, chiefly the small contracted liver. Some of the patients had ascites. Thirty-seven observations were made; bilirubinemia was found in 31 instances, and in all the 31 cases there was jaundice. The bilirubinemia varied from 20 to 350 . Bilirubin was found in the urine of 17 patients, but there was a marked disparity between cholemia and choluria; six plasmas were found at 60 or over without choluria; bilirubin was not withheld from the lymph, but was not always freely yielded to the kidney. In none of these cases was there any great impairment of kidney function, so the bilirubin is probably fixed to the plasma. In the cases in which the jaundice had been the most prolonged this fixation was most marked; the severity of the systemic symptoms was never an index to the fixation. Bile salts were found in the blood in 10 cases; in the urine in 12 . There seems to be no retention of the salts in the blood.

There is in this group a very common and mystifying dissociation of the biliary elements in both blood and urine, from which the observer has gathered nothing but confusion. In two cases there was at one time a marked increase in the bile output found in the stool, associated with a marked jaundice and deep choluria, showing that the formation of bile pigment had been increased. In three cases there was a vary marked diminution of the output of bile in the stool simultaneously with a want of bile in the urine and a very moderate jaundice, showing a definite diminution of the formation of bile. In five there was a distinct diminution of bile in the stool at a time when the blood, urine and skin were deeply stained, thus showing that obstruction to the drainage of bile sometimes occurs. Several cases

3. Hooper and Whipple: Am. Jour. Physiol., 1916, 40, 332.

4. Hoover, C. F., and Blankenhorn, M. A.: Dissociated Jaundice, ThE Archives Int. Med., 1916, 18, 289. 
went through a period of jaundice and ascites without showing any demonstrable change in the daily output of bile in the stool.

Hanot's Cirrhosis.--Three cases of Hanot's cirrhosis were examined, but very little disturbance in the formation or distribution of bile could be found; a thing quite surprising considering the alteration in the size and consistency of the liver and the general symptoms. The same can be said of three cases of syphilitic disease of the liver, except of one in which there was great deformity about the hilus of the liver, as shown at necropsy, with obstruction of the ducts.

Obstructive Jaundice.-Nineteen cases of obstruction of the bile ducts were observed and twenty-three specimens of blood taken. Twenty-three specimens contained bilirubin, most of them over 100 . All were jaundiced, and all except two were accompanied by bilirubin in the urine. In this group there is very little disparity between cholemia and choluria, but inasmuch as there is a higher average cholemia than in the cirrhosis group, that point in difference is of little value. In twelve out of twenty-three plasmas, bile salts also were found. In the urine, too, bile salts and bilirubin were very seldom dissociated in this group. This consistency between bile pigment and bile salts in both blood and urine in obstructive jaundice, especially at the onset, or while the process is progressing, is regarded by me as characteristic of obstructive lesions. Very few exceptions to this have been found that cannot be explained by some complication, such as septicemia, nephritis, etc. After the obstruction is relieved the bile salts disappear from the urine and blood first, and we then have, of course, a dissociation, but the dissociation is of renal origin. The early obstructive lesions are the best and most common examples of the so-called "complete icterus": that is, bilirubin and bile salts in both urine and blood. The stool in obstructive jaundice varies in its bile content according to the extent and duration of the obstruction. That the bilirubin content of the blood and urine must necessarily become greater as the amount in the stool diminishes is not true. I observed three cases in which the stools were acholic for over a week, during which time the jaundice remained the same, and the same amount of bilirubin was given off each day by the urine; apparently the formation of bile had been diminished by the obstruction to an amount that could be eliminated by the kidney as fast as it was formed. In all three cases there was no way of estimating the condition of the portal blood flow, so that it is not certain that biliary obstruction alone was the inhibiting factor.

One case has been observed, including a necropsy, in which no lesion save obstruction could be found, yet the patient went six days with only a very slight increase in the cholemia and jaundice. I am 
of the opinion that under some circumstances obstruction alone will inhibit the formation of bile.

Sixteen cases were investigated in which a diagnosis of cholelithiasis was made, but in which no obstruction could be proved. Seven of the plasmas contained bilirubin and three bile salts, and that only in very small amounts. None of the patients was jaundiced and none had bile in the urine (either pigment or salts). Seven of these patients were operated on, and in three gallstones were found; but of the three only one showed cholemia before the operation, while of the four who had no gallstones at operation, three had shown cholemia before the operation. The examination of the blood for small amounts of bile has thus far been of no aid in the diagnosis of gallstones that are producing no obstruction.

Catarrhal Jaundice.-Seven cases of catarrhal jaundice were examined and nine specimens of blood taken. Bilirubin was found in all; bile salts in four. The distribution and behavior of the bile resembled obstructive jaundice in this respect: there was little dissociation of the pigments and salts in both blood and urine except after the process began to subside. There was also an intestinal hypocholia. Unlike most obstructive lesions, there was frequently urobilin in the urine.

Chronic Passive Congestion.-Twelve cases of heart disease with chronic passive congestion of the liver were examined; most of them while decompensated. Bilirubin was present in ten cases; bile salts in three. Only small amounts of bilirubin were found, and but two of the patients were jaundiced, and only one had bile in the urine. There was considerable dissociation in the blood. Like Hanot's cirrhosis, there is little change in the distribution of the bile pigment in proportion to liver changes.

Septicemia.-Thirteen patients with septicemia were investigated, including 3 cases of typhoid, 5 of streptococcus and 4 of colon infections. In 10 there was bilirubin in the plasma and 5 bile salts; 6 had large amounts and were deeply jaundiced, but only 2 of the 6 had bile salts in the blood. On account of the liability to so many complications, especially nephritic, it is impossible to generalize in this small group.

Pneumonia.-Forty cases of pneumonia were investigated and 48 specimens examined. Thirty specimens contained bilirubin, averaging 50 , the maximum being $100 ; 14$ contained bile salts; 12 patients were jaundiced and 6 had bile in the urine; 19 had urobilin in the blood and 39 in the urine. It is this group that provided the large number of specimens with urobilin in the blood, but in only those cases in which the specimen was taken early in the course was the urobilin found. Urobilin was found to appear in the blood very early in the course of 
the disease - in several instances preceding jaundice. It also disappears very rapidly - from the blood several days to a week before it is absent from the urine. In but four instances was urobilin found on two successive days. The severity of the infection was not an apparent factor on two successive days. The severity of the infection was not an apparent factor in the presence of urobilinemia: out of 15 patients who showed it, ten recovered.

Examination of the stool in pneumonia cases showed an increase in bile output for several days; usually during the period of urobilinemia or jaundice.

Malaria.-Five cases of malaria were examined and six specimens taken. Five out of six contained bilirubin, and none contained bile salts. The absence of bile salts is significant, but the numbers are too small to justify much speculation. The output of bile in the stool in three of this group was increased; in the other two no measurements were taken.

Ectopic Pregnancy.-Three cases of ectopic pregnancy were found and four specimens examined. Bilirubin was found in all four specimens, but only in small amounts; none of the patients was jaundiced. Bile salts were present in but one. The stools showed no increase in bile output.

Teniasis.-Three cases of Dibothriocephalus taeniasis were found and four specimens examined. Bilirubinemia was present in all, but only one patient was jaundiced. Bile salts were present in the plasma of two out of the three patients. In all three the stools showed an increase in bile. This group cannot be distinguished from primary anemia by the behavior and distribution of bile.

Plumbism.-Four cases of chronic lead poisoning were found and three patients had bilirubin in the plasma, but all four had bile salts in the plasma. Bilirubin was present in small amounts and none of the group of patients was jaundiced. The urine in all was negative; the stools also. The presence of bile salts in this group may be of some importance.

Primary Anemia. - Thirty-four cases of primary anemia were examined and 42 specimens taken. Thirty-two specimens of plasma contained bilirubin and 13 of the patients were jaundiced. Bilirubin was not found in the urine of any. The bilirubin in the blood in 10 specimens was 50 or over. Bile salts were found in 24 plasmas, and in 2 urines. Urobilin was found in 20 urines; the stools showed increase in bile output in all that were satisfactorily investigated. This group is characterized by a particularly high degree of cholemia without jaundice or choluria, and by a marked retention of bile salts in the blood. This is essentially the same as previously reported 
Recently it has been found that in primary anemia there is a marked impairment of kidney function, and the possibility of the choluria in primary anemia being due to renal impairment must be considered. I therefore examined the plasma of 16 nephritics to determine somewhat the rôle of renal impairment in the accumulation of bile in the blood. These plasmas are uncommonly clear and colorless. Only 2 contained bilirubin; one at 5 , and one at 10 . This in some cases can be explained by the retention of water in the plasma. Seven specimens contained bile salts. None of the patients were jaundiced and the urine in all was negative. It is quite significant that 7 patients without apparent cause had increased amounts of bile salts in the blood, and it is very probable that the renal impairment in primary anemia is an important factor in the accumulation of the salts in the blood.

Secondary Anemia.-Seven cases of severe secondary anemia were examined. The plasma in six patients contained very small amounts of bilirubin, but none contained bile salts. Four of these patients were thought to be jaundiced, but this is explained by the great pallor. The urine of all was negative; the stools of all this group that were satisfactorily investigated showed a slight diminution of bile output. This fact is significant only when we compare this group with the primary anemias due to Dibothriocephalus.

The plasma of twenty-two perfectly normal persons was examined and thirty-four specimens taken. Eight specimens contained bilirubin and four bile salts. The urine in all was negative. In connection with Dr. Christie, who has examined the blood recently of many normal persons in his work on renal function, an opportunity was given me to obtain a limited number of specimens at intervals during the day, in relation to taking food and abstaining from food, but thus far the material is too limited to be of value, except that nothing radically contradictory has been found. 